\title{
HIV Prevention and Health Poverty Alleviation - Liangshan Prefecture, Sichuan Province, China, 2017-2020
}

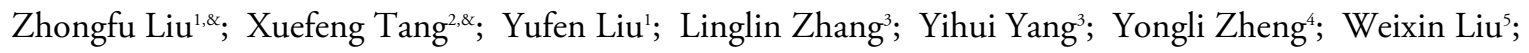 \\ Chunnong Jike ; Hong Mai'; Heying Fan ${ }^{8}$; Gang $\mathrm{Yu}^{6}$; Mengjie Han ${ }^{1, * *}$; George F. Gao ${ }^{9, *}$
}

\section{BACKGROUND}

Liangshan, officially the Liangshan Yi Autonomous Prefecture, is an autonomous prefecture occupying much of the southern portion of Sichuan Province. It has been a remote and deeply impoverished area and also has the largest population of ethnic Yi people in China (1). For many years, due to geographical environment conditions, traffic conditions, and other historic restrictions, the human immunodeficiency virus (HIV) epidemic in Liangshan Prefecture has become the focus of HIV prevention and control in China since the first acquired immunodeficiency syndrome (AIDS) case was reported in this area in 1995 (2-3). Since then, newly reported cases of HIV/AIDS in Liangshan Prefecture have been rising in number every year, especially in 2006, 2007, and 2009 , the newly reported number of HIV/AIDS cases increased exponentially over the previous years. In 2008, Butuo County of Liangshan Prefecture became the first county in China to report that living HIVinfected persons and AIDS patients accounted for over $1 \%$ of the resident population. In 2009, 2012, and 2013, Zhaojue, Yuexi, and Meigu counties, respectively, reported an HIV prevalence of over $1 \%$. Efforts have been made at all levels to carry out HIV prevention and control, and some progress has been made. However, the local HIV epidemic has not been effectively controlled. In order to protect the people's health and promote poverty alleviation, the National Health Commission and Sichuan Provincial Government jointly issued the "First Phase Action Plan for HIV Prevention and Health Poverty Alleviation in Liangshan Prefecture, Sichuan Province, China (2017-2020)" (hereafter referred to as the "Action Plan"). Focusing on four key counties of Butuo, Zhaojue, Yuexi, and Meigu (hereafter referred to as the "four key counties"), a special campaign against HIV and poverty in Liangshan Prefecture was launched in September 2017.
After three years of effort, the initiatives implemented in this area have markedly improved the health of local residents, and contributed to local poverty alleviation. The purpose of this article is to summarize the key strategies and measures implemented, demonstrate the changes of core indicators of HIV prevention and control, and describe the contribution to local poverty alleviation, providing important reference for AIDS responses in other areas of China.

\section{MAJOR STRATEGIES \& MEASURES IN PRACTICE}

Over the past three years since the implementation of the Action Plan, a series of strategies and measures have been implemented in Liangshan Prefecture. The local government and residences carried out all-round health education, conducted prevention of mother-tochild transmission (PMTCT), strengthened HIV screening, and implemented standard treatment of people living with HIV/AIDS. Moreover, they enforced drug control and rehabilitation, focused on HIV intervention among key populations, and aimed to mobilization of social forces to promote poverty alleviation through healthcare.

\section{Health Education}

The local government and its people have continuously searched for new and innovative ways to provide HIV/AIDS health education, mainly based on local sociocultural characteristics of the Yi ethnic group. It focused on promoting the formation of "active prevention" environments and advocated a healthy and civilized lifestyle. According to characteristics of different populations and ethnic minorities' customs, information, education, and communication (IEC) materials with bilingual and easy-to-understand language were developed to ensure full coverage of publicity and education. During the 
"World AIDS Day", "International Day Against Drug Abuse and Illicit Trafficking", and the Yi Ethnic Festival, the local government made every effort to advance publicity and education on HIV prevention. It expanded the combination of regular and centralized health promotion to guarantee effectiveness, proposing that publicity on HIV prevention be combined with activities such as poverty alleviation, health literacy promotion, establishment of a "drug-free families" campaign, and rural activities for culture, science, and technology. In addition, professional publicity teams composed of those who understood local culture and knew the Yi language well were set up to carry out face-to-face education through home visits (4). Also, these teams used schools as the primary site for health education to comprehensively improve health literacy of the new generation. Sexual ethics, sexual responsibility, and safe sexual behaviors became the focus of education and were included in the training of HIV prevention in schools at all levels. In 2020, the public awareness rate of HIV-related knowledge was $95.84 \%$, which increased by $61.22 \%$ compared with the baseline $(34.62 \%)$ in 2017. Meanwhile, the awareness rate of HIV-related knowledge in 2020 among infected persons was $96.24 \%$, an increase of $39.63 \%$ compared with the baseline $(56.61 \%)$ in 2017. In addition, the number of high-risk groups that had multiple sexual partners and did not use condoms decreased. Patient medication compliance was also greatly increased.

\section{Expansion of HIV Testing}

In an attempt to maximally identify people living with HIV/AIDS, HIV testing was carried out among people with 18 months of age and above in free health check-ups for people in 8 counties severely affected by HIV in 2018, including the 4 key epidemic counties that had HIV prevalence over $1 \%$ among local residents and 4 other counties (Puge, Jinyang, Xide, and Ganluo). Meanwhile, provider-initiated HIV testing and counseling (PITC) was conducted among $100 \%$ of hospital inpatients, and $70 \%$ of outpatients in departments such as obstetrics and gynecology, dermatology, and urology of medical institutions at all levels in the 4 key counties. In addition, the informed and non-refusal HIV testing services among women of childbearing age was intensified, and free pre-marital healthcare, HIV testing for pregnant women in their first visit, and re-testing for pregnant women before childbirth were also implemented. Also, the grassroots HIV prevention teams in township health centers guided village-level health workers to mobilize HIV testing. Village doctors provided a pregnancy testing service for HIV-infected women of childbearing age and helped mobilize and send them to medical institutions to receive healthcare services during pregnancy. All detainees in detention centers, such as drug maintenance treatment clinics, drug rehabilitation centers, and prisons, were tested for HIV on entry into the facilities.

\section{Treatment}

The four key county CDC and township health centers established a management system for tracking people living with HIV/AIDS. Once there were newly found untreated patients in the CDC or other medical institutions, they would be promptly referred to local antiretroviral treatment (ART) center for treatment. In this process, the strategy of "First diagnosis by medical institutions, seamless referral by CDC, timely treatment by designated medical institutions, and follow-up management by village health center" was put in place to avoid missing cases and ensure quality of treatment (5-6). Meanwhile, in order to achieve full coverage of HIV prevention and treatment services and precise management, the " $1+\mathrm{M}+\mathrm{N}$ " triangle model was set up based on townships. "1" represented the responsible person in charge of township government who was also the first person in charge of HIV prevention and control in the town; " $\mathrm{M}$ " signified township responsible persons and health workers; and "N" was composed of village doctors, village health workers, and village maternal and child workers. In this model, several local innovative approaches were explored. The first one was called "Budi-ri". As local people did not know what viruses and bacteria were, and they called the microorganisms that were harmful to their health "Budi-ri". In the " $1+\mathrm{M}+\mathrm{N}$ " model, village health workers showed a cartoon of "Budi-ri" and vividly demonstrated changes on the number of "Budi-ri" under standardized medication, nonstandard medication, and no medication to the patients, which was of great help to the treatment work. The second was the practice of the "from mouth to stomach" strategy. In order to ensure correct and standardized medication and prevent treatment failure, village health workers delivered medicine to the patients' homes, watched them take medicine, let them open their mouth after swallowing to ensure that the medication was consumed, and ensured the dosages were correct. The third one was called "color code 
management". According to the viral load testing results, cases of treatment failure were marked with "red", and treatment success were marked with "blue", which was convenient for classification management and helped to improve patients' cognition of their own treatment.

\section{Interventions Among Key Populations}

Condom use was vigorously promoted by carrying out training on condom use, and free condoms were put in places where people would always see them in waiting areas of health institutions. Among HIV serodiscordant couples, local health staff conducted face-to-face health education and condom use guidance every quarter, mobilized them to receive ART, and further strengthened patients' follow-up management. HIV testing for the negative spouse was conducted at least once a year and provided pre-exposure prophylaxis (PrEP) or post-exposure prophylaxis (PEP) services for those who were untreated or poorly treated. Meanwhile, it also strengthened comprehensive interventions on drug abuse, including standardization of methadone maintenance treatment (MMT) clinics management and improvement of compliance of drug users, maintaining treatment among drug addicts continuously. Extension points were set up in villages and towns in areas without maintenance treatment clinics, improving the accessibility of maintenance treatment. In addition, the connection between compulsory isolation and detoxification, community detoxification, community rehabilitation, and drug maintenance treatment was also further strengthened.

\section{Prevention of Mother-to-Child}

\section{Transmission}

During the implementation of the "Action Plan" prevention of mother-to-child transmission was further strengthened. It included HIV testing during premarital examinations and the provision of health consultations, healthcare services, pregnancy and contraception guidance for infected women of childbearing age to help avoidance of unwanted pregnancy and timely referrals for treatment. With the guidance of family planning services, early detection of pregnant women, timely delivery of maternal and child healthcare services, and HIV testing were conducted. Pregnant women with HIV infection were included in the high-risk population management, and were urged to receive regular pregnancy and childbirth healthcare, relevant auxiliary examinations, and hospital delivery.
Free hospital delivery services were provided for infected pregnant women, and standardized prevention and intervention services were provided for them and their newborns (7-8). Those waiting to give birth and pregnant women living in remote areas were encouraged to be hospitalized for delivery in advance. Additionally, it advocated "one-to-one" tracking of infants born to pregnant women, guiding them to take preventive ART drugs, feeding, and early diagnosis of HIV.

\section{MAIN OUTCOMES}

\section{Basic Changes}

In 2019, there were 4,694 and 1,964 newly reported HIV-infected persons and AIDS patients in Liangshan Prefecture and the four key counties, decreasing $15.73 \%$ and $37.33 \%$ compared with that in 2017 , respectively, and had an average annual reduction rate of $8.90 \%$ and $26.32 \%$, respectively. The proportion of newly reported cases of 4 key counties in the whole prefecture decreased from $56.27 \%$ in 2017 to $41.84 \%$. By the end of 2019, there were 24,663 survivals of HIV/AIDS in the 4 key counties, accounting for $59.40 \%$ of the whole prefecture, with a decrease of $5.11 \%$ compared with that in 2017 . As of 2019 , the number of people involved in HIV prevention and control was 3.67 times that of 2017. The number of HIV-infected persons per capita of health workers was reduced from 21.98 in 2017 to 6.52 in 2019. From 2017 to 2019, AIDS funds from the central government, Sichuan Province, and Liangshan Prefecture increased year by year. The continuous investment of funds has comprehensively promoted the construction of medical and health service systems and the cultivation of health personnel teams at the prefecture, county, township, and village levels. It has also advanced the information construction and wholeprocess traceability of drug management and supplemented the shortcomings of infrastructure and medical equipment of grass-roots medical and health institutions. The basic changes including the number of infected persons, AIDS funds, and number of health staff engaged in the AIDS response were shown in detail in Table 1.

\section{Changes of Core Indicators}

By the end of 2020, the proportion of people living with HIV who knew their HIV status, the proportion of people with diagnosed HIV infection who received 
TABLE 1. Basic changes in Liangshan Prefecture and 4 key counties from 2017 to 2019.

\begin{tabular}{|c|c|c|c|c|c|c|c|c|}
\hline \multirow{2}{*}{ Indicator } & \multicolumn{4}{|c|}{ Liangshan Prefecture } & \multicolumn{3}{|c|}{ Four key counties } & \multirow[b]{2}{*}{2020} \\
\hline & 2017 & 2018 & 2019 & 2020 & 2017 & 2018 & 2019 & \\
\hline \multicolumn{9}{|l|}{ Basic information } \\
\hline Number of living infected persons & 34,964 & 38,545 & 41,524 & 43,697 & 22,553 & 24,224 & 24,663 & 25,534 \\
\hline Number of newly reported infected persons & 5,570 & 10,105 & 4,694 & 3,018 & 3,134 & 6,714 & 1,964 & 1,456 \\
\hline \multicolumn{9}{|l|}{ AIDS funds } \\
\hline Central government investment (100 million yuan) & 0.45 & 0.87 & 1.3 & 1.9 & 0.21 & 0.43 & 0.59 & - \\
\hline $\begin{array}{l}\text { Financial investment of Sichuan Province (100 million } \\
\text { yuan) }\end{array}$ & 0.14 & 0.72 & 0.77 & 0.08 & 0.02 & 0.31 & 0.28 & - \\
\hline $\begin{array}{l}\text { Financial investment of Liangshan Prefecture (100 } \\
\text { million yuan) }\end{array}$ & 0.19 & 0.21 & 0.22 & 0.22 & 0.05 & 0.02 & 0.02 & - \\
\hline \multicolumn{9}{|l|}{ Staff on AIDS response } \\
\hline $\begin{array}{l}\text { Number of people responsible for local management } \\
\text { (persons) }\end{array}$ & 404 & 504 & 575 & - & 105 & 188 & 195 & - \\
\hline Number of people engaged in " $1+\mathrm{M}+\mathrm{N}$ " system & 9,282 & 12,217 & 11,143 & - & 1,026 & 4,072 & 3,781 & - \\
\hline $\begin{array}{l}\text { Per capita number of infected persons managed by } \\
\text { " } 1+M+N \text { " system }\end{array}$ & 3.77 & 3.16 & 3.73 & - & 21.98 & 5.95 & 6.52 & - \\
\hline
\end{tabular}

Note: -: Data not available.

Abbreviation: AIDS=acquired immunodeficiency syndrome.

sustained ART, and the proportion of people receiving ART who had viral suppression reached 90.52\%, $94.81 \%$, and $94.80 \%$, respectively, which was an increase of $10.80 \%, 130.68 \%$, and $64.87 \%$, respectively; the mother-to-child HIV transmission rate, sero-discordant couples transmission rate, and HIV new infection rate decreased to $3.66 \%, 0.85 \%$, and $0.02 \%$, respectively, which was a decrease of $59.33 \%, 87.94 \%$, and $68.75 \%$, respectively. In the past three years, the HIV detection rate, ART coverage, and HIV viral suppression rate rose steadily; meanwhile, MTCT rate, transmission rate among serodiscordant couples, and HIV new infection rate decreased significantly. See Figure 1 and Table 2 for details.

\section{CHALLENGES \& PROSPECTS}

During the three-year period, positive changes of the main indicators on HIV prevention and control were seen in Liangshan Prefecture. The mechanism of HIV prevention and treatment has been improved, and primary medical services have been elevated; in addition, major breakthroughs have been made in important and difficult tasks such as treatment, PMTCT, and HIV prevention among key populations

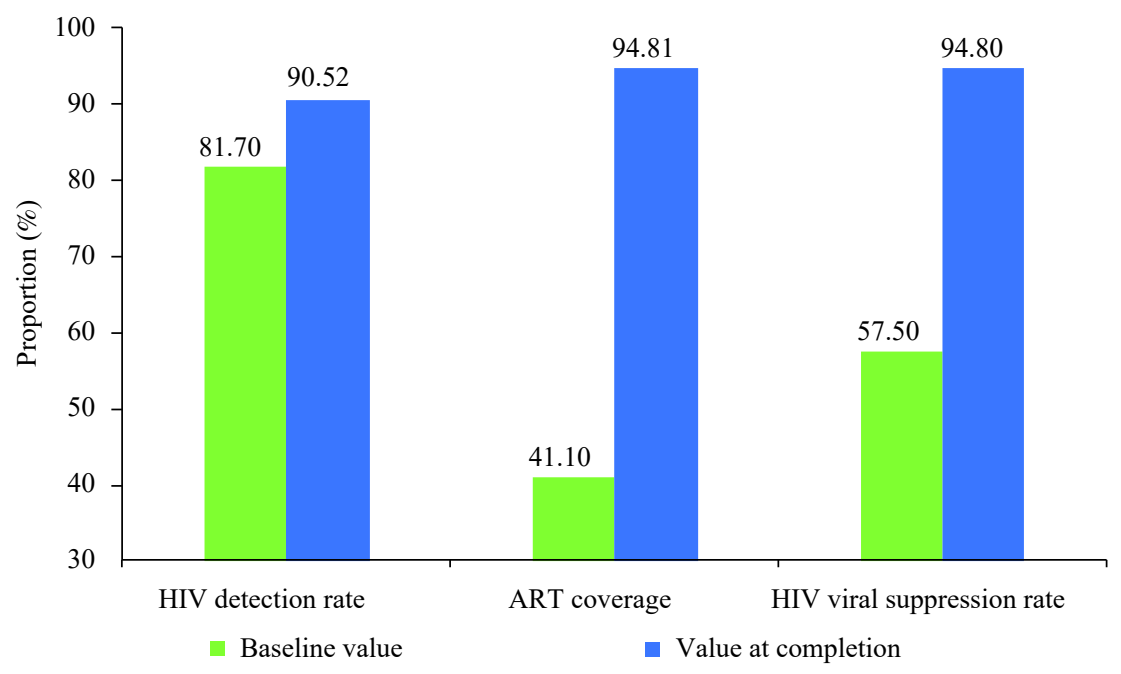

FIGURE 1. Changes of HIV detection rate, ART coverage, and HIV viral suppression rate between baseline and completion. Abbreviations: HIV=human immunodeficiency virus; ART=antiretroviral treatment. 
TABLE 2. Changes of MTCT rate, HIV transmission rate among sero-discordant couples, and HIV new infection rate from 2017 to 2020, Liangshan Prefecture.

\begin{tabular}{lcc}
\hline \multicolumn{1}{c}{ Indicators } & $\begin{array}{c}\text { Baseline } \\
\text { value }\end{array}$ & $\begin{array}{c}\text { Value at } \\
\text { completion }\end{array}$ \\
\hline MTCT rate (\%) & 9.00 & 3.66 \\
HIV transmission rate among sero- & 7.05 & 0.85 \\
discordant couples (\%) & 0.64 & 0.20 \\
HIV new infection rate (\%) &
\end{tabular}

Abbreviations: MTCT=mother-to-child transmission;

$\mathrm{HIV}=$ human immunodeficiency virus.

(9). In fact, Liangshan Prefecture's practice on HIV precision management and primary medical and health services provision not only ensured the effectiveness of local HIV prevention and treatment, but also provided an important reference for the prevention and control of major chronic infectious diseases at the grass-roots level in other similar areas in China.

At present, Liangshan Prefecture still encounters significant challenges in HIV prevention and control. For instance, although technical personnel have been trained in recent years, the local capacity of HIV prevention and control at the township and village levels needs to be further improved. And also, the assessment and evaluation system at the local level still needs to be refined. The evaluation methods for the workload and effectiveness of " 1 ," "M," and "N" still need to be adjusted and improved to better standardize and quantify their workload, so as to improve the evaluation systems for both the township and village levels (10). In addition, the sustainability of the current response needs to be considered and laid out. Strong government leadership, participation of the whole society, and financial support were the "engine" of the sustainable development of AIDS response; however, due to different situations of villages and towns and different factors affecting work sustainability, the current response needs to be gradually adapted in line with the needs of different scenarios of areas, so as to maintain its overall sustainability.

In summary, Liangshan Prefecture's efforts have been seen as China's best practice on the consolidation of "four-party responsibilities" of the government, departments, society, and individuals. Looking to the years ahead, efforts should be continuously made to further boost the achievements for further strengthening AIDS response and controlling the HIV epidemic effectively. Also, the instructive exploration on the grassroots AIDS response in this area is worth learning, popularizing, and applying in other severely affected HIV-epidemic areas in China. doi: $10.46234 / \mathrm{ccdcw} 2021.250$

\# Corresponding authors: Han Mengjie, mjhan@chinaaids.cn; George F.Gao,gaofu@chinacdc.cn.

\footnotetext{
${ }^{1}$ National Center for AIDS/STD Control and Prevention, China CDC, Beijing, China; ${ }^{2}$ Sichuan Provincial Center for Disease Control and Prevention, Chengdu, Sichuan, China; ${ }^{3}$ Sichuan Provincial Center for Disease Control and Prevention, Chengdu, Sichuan, China; ${ }^{4}$ Chengdu Public Health Clinical Center, Chengdu, Sichuan, China; 5 Sichuan Provincial Maternity and Child Health Care Hospital, Chengdu, Sichuan, China; ${ }^{6}$ Liangshan Prefecture Center for Disease Control and Prevention, Xichang, Sichuan, China; ${ }^{7}$ The First People's Hospital of Liangshan Prefecture, Xichang, Sichuan, China; ${ }^{8}$ Liangshan Prefecture Maternal \& Child Health and Family Planning Service Center, Xichang, Sichuan, China; ${ }^{9}$ Chinese Center for Disease Control and Prevention, Beijing, China.

${ }^{\&}$ Joint first authors.
}

Submitted: November 10, 2021; Accepted: November 21, 2021

\section{REFERENCES}

1. Liu TT, Li YH, Li YB, Jin XZ, Wang XL. Investigation and suggestions to AIDS health education in rural areas of Liangshan Yi Autonomous Prefecture. Chin J Health Educ 2010;26(5):334 - 7. http://dx.doi.org/ 10.16168/j.cnki.issn.1002-9982.2010.05.016. (In Chinese).

2. Wang DJ, Chen DD, Cui HH, Zhang Y. Poverty status and influencing factors of AIDS patients, Liangshan Yi nationality area. Mod Prev Med 2019;46(3):389-93. https://kns.cnki.net/kcms/detail/ detail.aspx? dbcode $=$ CJFD $\&$ dbname $=$ CJFDLAST2019\&filename $=$ XDYF201903002\&uniplatform=NZKPT\&v=qRZsO58PZZTw87B29 XBAZ3NKuJmpq2QSaC6HotuJAt5QkoA4D_a0rGZf7XtsCWpD. (In Chinese)

3. Yang SJ, Luo M, Zhang SH, Yao YN, Wang QX, Liao Q. Overview on culture and customs related to AIDS epidemic and prevention among Yi people in Liangshan. Chin J AIDS STD 2017;23(3):271 - 4. http:// dx.doi.org/10.13419/j.cnki.aids.2017.03.28. (In Chinese)

4. Liu TT, Li YB, Jiang H, Jiang S, Yang C, Huang XG, et al. Targeted exploration of AIDS prevention publicity and health education in Liangshan. Chin J AIDS STD 2020;26(9):915 - 7. http://dx.doi.org/ 10.13419/j.cnki.aids.2020.09.01. (In Chinese).

5. Zhou C, Liang S, Li YP, Yang Y, Liao LJ, Xing H, et al. Influencing factors on the death of HIV/AIDS patients treated with antiviral treatment in Butuo county, Liangshan Yi Autonomous Prefecture, 2010-2019. Chin J Epidemiol 2021;42(5):886 - 90. http://dx.doi.org/ 10.3760/cma.j.cn112338-20200902-01115. (In Chinese).

6. Zhuo MLC, Zeng YL, Huang J, Wu M, Dong CT, Yu H, et al. Evaluation of the timeliness and the effect of anti-retroviral treatment on children infected with HIV in Butuo county. J Prev Med Inf 2021;37(7):905-10. https://d.wanfangdata.com.cn/periodical/yfyxq bzz202107006. (In Chinese)

7. Zhang G, Liu P, Qu MX, Liu TT, Tian CH, Lai WH, et al. Effect of technical support and capacity building for prevention of mother-tochild transmission of AIDS in four counties of Liangshan prefecture. Chin J AIDS STD 2020;26(8):797 - 800. http://dx.doi.org/10.13419/ j.cnki.aids.2020.08.01. (In Chinese)

8. Liu P, Kang WT, Zhang G, Liu TT, Wu JM, Peng J, et al. Outcomes of pilot project of prevention of mother-to-child transmission of HIV/AIDS in 10 townships of 4 counties in Liangshan prefecture. Chin J AIDS STD 2020;26(10):1033 - 5. http://dx.doi.org/10.13419/j.cnki. aids.2020.10.01. (In Chinese).

9. Xiong W, Wu QH. Liangshan Prefecture has made remarkable achievements in AIDS respones. China Rural Health 2020;12 (23):5 - 6. http://dx.doi.org/10.3969/j.issn.1674-361X.2020.23.002. (In Chinese)

10. Han JY, Yang SJ, Pei R, Jike CN, Gao B, Yu G, et al. Establishment of an AIDS risk assessment index system based on social determinants of health, Liangshan. Mod Prev Med 2019;46(7):1224-8. https://d. wanfangdata.com.cn/periodical/xdyfyx201907018. (In Chinese). 